\title{
Kalirin Dbl-Homology Guanine Nucleotide Exchange Factor 1 Domain Initiates New Axon Outgrowths via RhoG-Mediated Mechanisms
}

\author{
Victor May, ${ }^{2}$ Martin R. Schiller, ${ }^{1}$ Betty A. Eipper, ${ }^{1}$ and Richard E. Mains ${ }^{1}$ \\ ${ }^{1}$ Department of Neuroscience, University of Connecticut Health Center, Farmington, Connecticut 06030, and \\ 2Department of Anatomy and Neurobiology and Department of Pharmacology, University of Vermont College of Medicine, \\ Burlington, Vermont 05404
}

The large multidomain Kalirin and Trio proteins containing dual Rho GTPase guanine nucleotide exchange factor (GEF) domains have been implicated in the regulation of neuronal fiber extension and pathfinding during development. In mammals, Kalirin is expressed predominantly in the nervous system, whereas Trio, broadly expressed throughout the body, is expressed at a lower level in the nervous system. To evaluate the role of Kalirin in fiber initiation and outgrowth, we microinjected cultured sympathetic neurons with vectors encoding Kalirin or with Kalirin antisense oligonucleotides, and we assessed neuronal fiber growth in a serum-free, satellite cell-free environment. Kalirin antisense oligonucleotides blocked the continued extension of preexisting axons. Kalirin overexpression induced the prolific sprouting of new axonal fibers that grew at the normal rate; the activity of Kalirin was entirely dependent on the activity of the first GEF domain. KalGEF1-induced sprouting of new fibers from lamellipodial structures was accompanied by extensive actin cytoskeleton reorganization. The kalGEF1 phenotype was mimicked by constitutively active RhoG and was blocked by RhoG inhibitors. Constitutively active Rac1, RhoA, and Cdc42 were unable to initiate new axons, whereas dominant-negative Rac1, RhoA, and Cdc42 failed to block axon sprouting. Thus Kalirin, acting via RhoG in a novel manner, plays a central role in establishing the morphological phenotypic diversity that is essential to the connectivity of the developing nervous system.

Key words: Kalirin; Trio; guanine nucleotide exchange factor; Dbl-homology; Rho GTPase; neuronal fiber outgrowth
The initiation, elongation, and guidance of emerging neuronal fibers in the developing nervous system require the integration and interpretation of extracellular signals (Tessier-Lavigne and Goodman, 1996; Luo et al., 1997; Hall, 1998; Suter and Forscher, 1998; Mueller, 1999; Luo, 2000; Dickson, 2001). After neuronal fiber initiation the lamellipodia and filopodia of advancing growth cones survey the environmental signals and pilot the elongating fibers as they navigate through attractant and repulsive cues. The extracellular signals and dynamic mechanisms of filamentous actin and microtubule assembly and disassembly have been studied in many cell types, but we have little understanding of how these processes are harnessed to generate diverse neuronal phenotypes.

Recently, genetic analyses of neuronal fiber elongation have identified roles for the Drosophila receptor tyrosine phosphatase Dlar, Abelson tyrosine kinase (Abl), its tyrosine phosphorylated substrate Ena, and the actin-binding protein profilin (Gertler et al., 1995; Krueger et al., 1996; Van Vactor, 1998). Furthermore, leukocyte antigen-related phosphatase (LAR) and Mena, their mammalian homologues, have been identified in filopodia and

Received Dec. 17, 2001; revised May 16, 2002; accepted May 28, 2002.

This work was supported by National Institutes of Health Grants DA-00266 and DK-32948 to B.A.E. and R.E.M. and HD-27468 to V.M. V.M. was Visiting Professor of Neuroscience at University of Connecticut Health Center. We thank Karen Braas for her support and expert preparation of the figures, Jianping Huang for plasmid preparations, Darlene D'Amato for getting the new lab running, and Jack Glaser and Kirsten Breggen of MicroBrightField Incorporated for advice in data analyses.

Correspondence should be addressed to Dr. Richard E. Mains, Department of Neuroscience, University of Connecticut Health Center, 263 Farmington Avenue, Room ARB 4047, Farmington, CT 06030. E-mail: mains@uchc.edu.

Copyright (C) 2002 Society for Neuroscience $0270-6474 / 02 / 226980-11 \$ 15.00 / 0$ focal adhesions (Gertler et al., 1995; Lanier and Gertler, 2000). Similarly, the interaction of p21-activated kinase (Pak) with the $\mathrm{SH} 2-\mathrm{SH} 3$ adaptor Dock is crucial for axon guidance (Hing et al., 1999).

Actin assembly requires the coordination of Rho family small GTPases, in which cycling between active GTP-bound and inactive GDP-bound states is regulated in part by guanine nucleotide exchange factors (GEFs) of the Dbl family. Among the Rho GTPases identified to date, Rac1, RhoA, and Cdc42 have been studied the most as selective molecular switches governing actin polymerization (Van Aelst and D'Souza-Schorey, 1997; Hall, 1998). Rac1, RhoA, and Cdc42 have prominent roles in growth cone formation, collapse, and steering, with evidence for cell type-specific responses (Nobes and Hall, 1995; Kozma et al., 1997; Hall, 1998; Lin and Greenberg, 2000). Expression of constitutively active or dominant-negative forms of Rho GTPases has resulted in axon guidance defects in Drosophila and Caenorhabditis elegans and altered fiber outgrowth in pheochromocytoma cells (Luo et al., 1997; Zipkin et al., 1997; Kaufmann et al., 1998; Hing et al., 1999). Recently, RhoG, acting via Rac1 and Cdc42, has been shown to stimulate PC12 neurite extension (Katoh et al., 2000).

Multiple Dbl family GEFs are expressed in the nervous system. Ephrin A, acting via its receptor, alters the specificity of the associated Dbl family member, ephexin, to cause growth cone collapse (Shamah et al., 2001). Kalirin and Trio, high-molecularweight proteins with dual Rho GEF domains (Fig. 1), seem to play unique roles in regulating process outgrowth (Steven et al., 1998; Awasaki et al., 2000; Bateman et al., 2000; Liebl et al., 2000; Newsome et al., 2000; Penzes et al., 2001a,b). Rat Kalirin (rKa- 


\section{Forms of Kalirin and Trio}

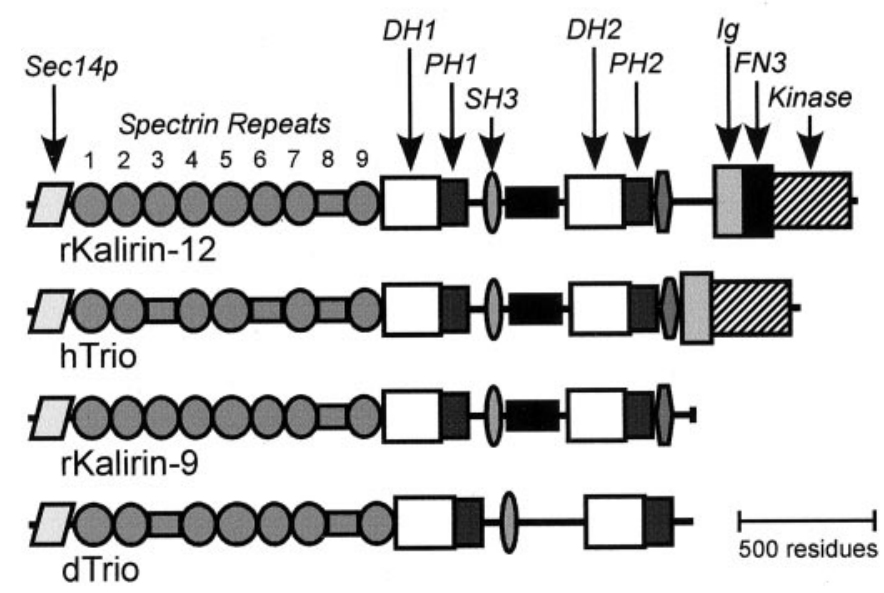

Figure 1. Kalirin and Trio. The structures of two isoforms of rat Kalirin (rKalirin-12; accession number AF232669; rKalirin-9; accession number AF232668), human Trio (hTrio; accession number AAC34245), and dTrio (accession number AB035419) are drawn to scale. Kalirin and Trio possess two distinct Dbl-homology (DH)/pleckstrin-homology (PH) domains typical of GEFs for Rho GTPases along with a putative serine/ threonine kinase domain. In addition, both hTrio and rKalirin possess a Sec14p domain, multiple spectrin-like repeats, $\mathrm{SH} 3$ domains, and $\mathrm{Ig}$ / fibronectin III (FN3) domains. Among the Kalirin isoforms that arise from alternative splicing, Kalirin-12 protein is structurally similar to hTrio. Kalirin-9, like dTrio, is devoid of the kinase domain; Kalirin-7 lacks the second GEF domain, and GEF1 is followed instead by a C-terminal postsynaptic density-95/discs large/zona occludens-1-binding motif mediating Kalirin-7 enrichment in postsynaptic density fractions (Penzes et al., 2000, 2001).

lirin) was identified as a binding partner to the cytoplasmic domain of peptidylglycine $\alpha$-amidating monooxygenase (PAM), a membrane protein essential to the biosynthesis of many neuropeptides (Alam et al., 1996, 1997). Human Trio (hTrio) was identified via its interaction with the intracellular phosphatase domains of human LAR (Debant et al., 1996).

Because the GEF domains of Kalirin and Trio interact with Rac1 and RhoA, both have the potential to coordinate attractant and repulsive cues. Unlike mammalian Trio, which has a broad tissue distribution, Kalirin is expressed preferentially in the adult nervous system (Johnson et al., 2000; Ma et al., 2001). Sympathetic neurons have been well studied with respect to neuronal fibers. In this work we assessed the role of Kalirin in postnatal sympathetic neurons, and we demonstrate a central role of Kalirin in the initiation of new axons and in axon outgrowth, mediated by RhoG, but not acting solely via Rac1 or Cdc42.

\section{MATERIALS AND METHODS}

Cell culture. Primary superior cervical ganglion (SCG) neuron cultures were prepared as described previously (May et al., 1995; Braas and May, 1999). Donor neonatal rats were decapitated, and the SCGs from three to four litters (typically 30-40 pups; 60-80 ganglia) were dissociated enzymatically to produce a pooled population of cells. Cells were plated at a density of $1.5 \times 10^{3}$ neurons $/ \mathrm{cm}^{2}$ onto rat tail collagen-coated $60 \mathrm{~mm}$ culture plates, treated with $10 \mu \mathrm{M}$ cytosine $\beta$-D-arabinofuranoside to remove non-neuronal cells, and maintained in defined serum-free medium containing $50 \mathrm{ng} / \mathrm{ml}$ nerve growth factor (Becton Dickinson Labware, Bedford, MA).

Kalirin vector constructs and microinjection. N-terminal myc-tagged Kalirin-12, Kalirin-9, kalGEF1, kalGEF2, kalKinase, and kal9DGEF1 were expressed in pEAK10.His (Edge Biosystems, Gaithersburg, MD). Kal9DGEF1 was constructed by deleting amino acid residues 1281-1583 of Kalirin-9 and joining the two fragments with a single Gly residue linker. All constructs were verified by sequencing. Transfection of Kalirin expression vectors induced lamellipodia formation and altered the phenotype of several fibroblast cell lines (Mains et al., 1999; Penzes et al., 2000). The kal9 $\Delta$ GEF1 construct yielded a protein $35 \mathrm{kDa}$ smaller than Kalirin-9. Fibroblasts expressing kal9 $\Delta$ GEF1 had the same phenotypes as fibroblasts expressing kalGEF2. Plasmids encoding RhoA, Rac1, and Cdc42 and constitutively active Rac1-Q61L and RhoA-Q62L plasmids were kind gifts from Dr. Richard Cerione (Cornell University, Ithaca, NY), Rac1-T17N and RhoA-T19N plasmids were generous gifts from Dr. Silvio Gutkind (National Institute of Dental Research, National Institutes of Health, Bethesda, MD), and Cdc42-Q61L and Cdc42-T17N constructs were obtained from A. Ghosh (Johns Hopkins University, Baltimore, MD). Expression vectors encoding enhanced green fluorescent protein-RhoG [EGFP-RhoG (G12V)], EGFP-RhoG (F37A), and RhoGIP122 were greatly appreciated gifts from A. Blangy (Centre de Recherche en Biochimie Macromoleculaire, Centre National de la Recherche Scientifique, Montpellier, France). The EGFP plasmid pEGFP-N2 was from Clontech (Palo Alto, CA); expression plasmids for Rac2 and RhoG were from the Guthrie cDNA Resource Center (Sayre, PA). All Rho GTPase and Kalirin expression vectors were tested first for their ability to alter $\mathrm{CHO}$, NIH 3T3, or pEAK Rapid cell morphology in transient transfection assays. Kalirin antisense oligonucleotide included TCA AAC CAT CAT TCC GAA AAG ATC C [nucleotides (nt) 37-61; GenBank U88157], which has only a 14 of 25 nt match with the corresponding Trio nt sequence; the scrambled control oligonucleotides included ACT CAA TAC TAC CCT AAG GAA CTA C; CAT ACA ATC ATC CCT AAG AGA TCA C; and TGG GAC CTC CAC GTT TAT CTC TCC C.

SCG neurons after 5-9 d of culture were viewed under an inverted phase/fluorescence microscope with $20 \times$ objectives. Kalirin or Rho GTPase constructs $(50-200 \mathrm{ng} / \mu \mathrm{l})$, pEGFP-N2 (200 ng/ $\mu \mathrm{l})$, and Texas Red-conjugated dextran (3000 MW, $2.5 \mathrm{mg} / \mathrm{ml}$; Molecular Probes, Eugene, OR) were coinjected directly into the nuclei of SCG neurons at 95 $\mathrm{HPa}$ for $400 \mathrm{msec}$ via a Transjection 5246/InjectMan system (Eppendorf, Hamburg, Germany). After $24-72 \mathrm{hr}$ the fiber structure of EGFPexpressing neurons were viewed with GFP filters for fluorescence photo microscopy with $20 \times$ or $40 \times$ objectives (Nikon Eclipse TE300, Nikon, Melville, NY; SPOT RT, Diagnostic Instruments, Sterling Heights, MI). GFP-positive cells were photographed 24,48 , and $72 \mathrm{hr}$ after injection, and the cells were matched by an independent observer. Most cells photographed at earlier times were present at later times; the number of GFP-positive cells increased with time, presumably because of the gradual expression of GFP. Neuronal expression of Kalirin or Rho GTPases was verified by immunocytochemical staining after termination of the experiments. In neurons coinjected with Kalirin and EGFP plasmids, $\sim 50-75 \%$ of EGFP-expressing neurons demonstrated altered phenotype, which reflected variability in cellular Kalirin expression levels as assessed by immunofluorescence staining for the myc-epitope tag. Expression from the smaller kalGEF1 expression vector was more consistent; over a fourfold range (50-200 ng/ $\mu \mathrm{l} \mathrm{kalGEF1)}>90 \%$ of all EGFPpositive neurons coinjected with kalGEF1 demonstrated strong phenotypic changes. Plasmid concentrations (in moles) for microinjection were directly comparable in some experiments, but kalGEF1, Kalirin-9, and Kalirin-12 expression levels in individual neurons were not normalized; differences because of the relative contributions of intramolecular modulation of Kalirin-9 and Kalirin-12 GEF1 activity were not determined. Morphometric analyses were performed with Neurolucida (MicroBrightField, Colchester, VT); statistical analyses were performed with SigmaStat (SPSS Science, Chicago, IL).

Antibodies and Western blot analyses. Antisera for Kalirin have been described (Johnson et al., 2000; Penzes et al., 2000). The Trio antibody (CT35) was raised by immunizing rabbits with a 14-amino-acid peptide corresponding to the COOH terminus of Trio, Trio(3025-3038), conjugated to keyhole limpet hemocyanin with glutaraldehyde (Milgram et al., 1997). The Trio antibody was affinity purified by linking the same peptide to Affi-Gel 10 and was used at a dilution of 1:200. Western blot analyses were performed as described (Johnson et al., 2000). Tissues were extracted into radioimmunoprecipitation assay (RIPA) buffer (Johnson et al., 2000), and protein concentrations were determined by using the BCA reagent (Pierce, Rockford, IL). Commercial antisera were used to visualize Rac1, RhoA, Cdc42 (Transduction Laboratories, San Diego, CA), RhoG (Santa Cruz Biotechnology, Santa Cruz, CA), and tau (Roche, Indianapolis, IN).

Metabolic labeling. Cultures were labeled by using $\left[{ }^{35} \mathrm{~S}\right] \mathrm{Met}$ in $\mathrm{Met}^{-}$ defined serum-free medium $(1 \mathrm{mCi} / \mathrm{ml})$ and chased in Met-containing 
medium as described (Alam et al., 1997). After a 30 min pulse or an additional 120 chase, the cells were extracted in RIPA buffer with protease inhibitors. Cell extracts were immunoprecipitated with excess Kalirin or Trio antibody; after isolation on protein A-Sepharose the samples were eluted and fractionated on 5\% SDS-PAGE gels. Gels were dried with Amplify for fluorography.

Rho GTPase binding and RhoG activation assays. Binding assays were performed by using glutathione $S$-transferase-GTPase (GST-GTPase) fusion proteins and extracts from cells expressing exogenous kalGEF1. Rac2 and RhoG cDNAs were subcloned into pGEX6P vector for expression as GST fusion proteins. GST fusion proteins $(10 \mu \mathrm{g})$ immobilized on glutathione-Sepharose 4B resin $(25 \mu \mathrm{l})$ were incubated with cell extracts for $2 \mathrm{hr}$. Extracts were prepared from HEK-293 pEAK Rapid cells transiently transfected with pEAK10.HisMyc-GEF1 by using TMT buffer (20 mM Na- $N$-tris(hydroxymethyl)methyl-2-aminoethanesulfonic acid, $\mathrm{pH} 7.4$, containing $10 \mathrm{~mm}$ mannitol, $1 \%$ Triton X-100, and protease inhibitors) and diluted 22-fold into magnesium lysis buffer [containing (in mM) 25 HEPES, pH 7.5, $150 \mathrm{NaCl}, 10 \mathrm{MgCl}_{2}, 1$ EDTA plus $1 \% \mathrm{NP}-40$ and $2 \%$ glycerol] for binding reactions. After being washed, the beads containing bound proteins were boiled into SDS-PAGE sample buffer and analyzed by Western blot analysis with a myc-antibody.

For RhoG activation assays the RhoGIP122 cDNA was subcloned into pGEX6P, expressed as a GST fusion protein (GST-RhoGIP), and immobilized on glutathione-Sepharose 4B beads. Cell extracts were prepared from HEK-293 pEAK Rapid cells transiently cotransfected with EGFP-RhoG and PEAK10.HisMyc-GEF1 or pEGFP (negative control) or with EGFP-RhoG (G12V) (positive control), using Rho-binding lysis buffer [containing (in mM) 50 Tris, $\mathrm{pH} 7.2,500 \mathrm{NaCl}$, and $10 \mathrm{MgCl}_{2}$ plus $1 \%$ Triton X-100, $0.5 \%$ Na-deoxycholate, $0.1 \%$ SDS]. Extracts were applied to the GST-RhoGIP resin, and the bound, activated RhoG was analyzed by Western blot analysis with the use of a RhoG antiserum.

Actin visualization and immunocytochemistry. Kalirin/EGFP-expressing SCG neurons were fixed with $4 \%$ paraformaldehyde for $15 \mathrm{~min}$ at room temperature, permeabilized with $0.1 \%$ Triton X-100, and labeled with 1:1000 TRITC-phalloidin (Sigma, St. Louis, MO) to visualize filamentous actin. Immunocytochemical staining for Kalirin forms and mycepitope was performed by using standard methods (Braas and May, 1999; Penzes et al., 2000).

\section{RESULTS}

\section{Kalirin induces extensive new neuronal fiber outgrowth}

Sympathetic postganglionic neurons have been used widely as a model to study neuronal fiber outgrowth and guidance (Giger et al., 1998; Guo et al., 1998; Francis and Landis, 1999; TsuiPierchala and Ginty, 1999; Xu et al., 2000). The neurons are quite homogeneous morphologically, with large acentric nuclei and defined fiber structures. SCG neurons exhibit diverse receptor systems that respond to known guidance cues and can be maintained readily in vitro under defined serum-free conditions without confounding regulatory factors. Dissociated sympathetic neurons in vitro, which display a simple phenotype as visualized by injecting plasmid-encoding EGFP into individual cells, provide an ideal system by which to evaluate neuronal GEF function. Typically, within the first few days of culture one to three primary fibers emanate from the large neuronal soma (30 $\mu \mathrm{m}$ diameter), extending rapidly $(5-20 \mu \mathrm{m} / \mathrm{hr})$ to lengths of several millimeters (Fig. 2A). The fibers develop complexity by branching at distal regions of elongating fibers. Approximately one branch point develops per $500 \mu \mathrm{m}$ in fiber length; no additional fibers emerge from the cell soma even after extended times in culture.

Alternate splicing events generate multiple Kalirin isoforms (Fig. 1). Because Kalirin transcripts and proteins, corresponding to Kalirin-12 and Kalirin-9, can be identified in adult and neonatal SCG as well as in cultured sympathetic neurons (see below; McPherson et al., 2002), we evaluated their effects on the growth of neuronal fibers. Constructs encoding myc-epitope-tagged Kalirin-9 and Kalirin-12 were microinjected along with a plasmid encoding EGFP. Sympathetic neuron EGFP expression within 24
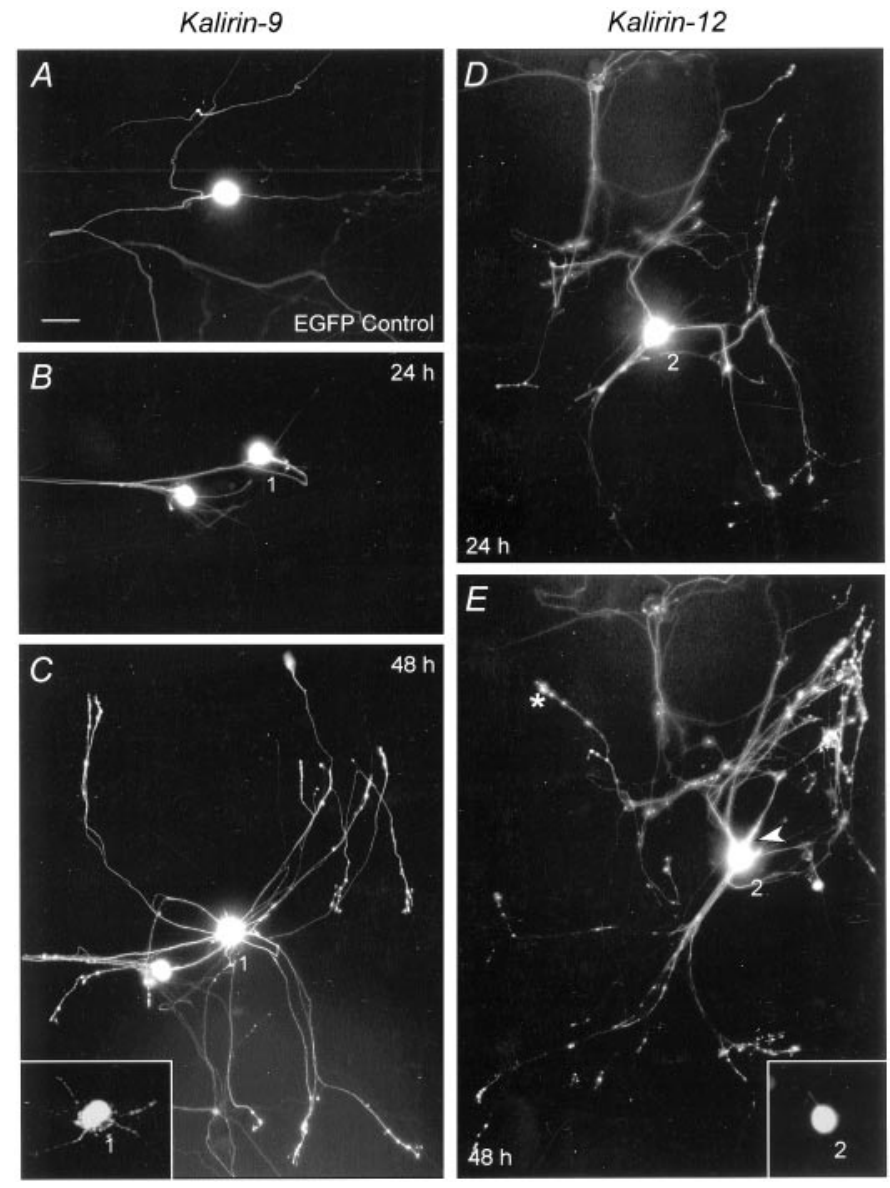

Figure 2. Kalirin initiates SCG fiber outgrowth. $A$, Control cultured SCG sympathetic neuron $72 \mathrm{hr}$ after microinjection with $200 \mathrm{ng} / \mu \mathrm{l} \mathrm{EGFP-}$ plasmid alone; two fibers project from the soma, with one branching a short distance from the soma. $B, C$, Sequential micrographs of neurons coinjected with EGFP and Kalirin-9 plasmid (200 ng/ $\mu$ l each). New fiber outgrowth is apparent when the 24 and $48 \mathrm{hr}$ postinjection micrographs are compared $(B, C)$. A burst of neuronal fiber initiation and extension is observed in Kalirin-9-expressing neurons between 24 and $48 \mathrm{hr}$. Cy3 immunofluorescence staining for the $m y c$-epitope was performed at the end of the experiment to demonstrate Kalirin-9 expression in neurons with fiber outgrowth (inset, cell 1). D, E, Sequential micrographs of a single neuron coinjected with EGFP and Kalirin-12 plasmids $(200 \mathrm{ng} / \mu \mathrm{l}$ each). Like for Kalirin-9, Kalirin-12 expression induced the outgrowth of new fibers and branches. After $24 \mathrm{hr}$ new fibers could be identified by bright EGFP fluorescent growth cones at distal fiber tips (asterisk). Comparison to the same Kalirin-12-expressing neuron $48 \mathrm{hr}$ after injection reveals new fibers (arrowhead) and a high density of growth cones emerging from extensive fiber branching. Kalirin-12 expression in the injected neuron with outgrowth phenotype was demonstrated by staining for myc-epitope; staining was confined to the soma (inset, cell 2).

hr allowed for visualization of both new and old processes, and photomicrographs of the same cells at 24, 48, and $72 \mathrm{hr}$ after injection allowed for a visual record of neuronal fiber development. Expression of Kalirin-9 (Fig. 2B,C) or Kalirin-12 (Fig. $2 D, E)$ produced a dramatic phenotype characterized strikingly by an initiation of multiple prominent new fiber outgrowths from the soma. This Kalirin-induced outgrowth of new fibers is uniquely different from the guidance functions ascribed previously to Drosophila Trio (dTrio) (Awasaki et al., 2000; Bateman et al., 2000; Newsome et al., 2000; Liebl et al., 2000). Compared with control cultures that were injected with the EGFP construct alone, the expression of exogenous Kalirin-9 or Kalirin-12 in- 
Table 1. Kalirin fiber analysis summary

\begin{tabular}{llll} 
& $\begin{array}{l}\text { Fiber } \\
\text { number }\end{array}$ & $\begin{array}{l}\text { Node } \\
\text { number* }\end{array}$ & $\begin{array}{l}\text { Segment } \\
\text { number }\end{array}$ \\
\hline Control $(n=24)$ & $1.9 \pm 0.1$ & $1.8 \pm 0.2$ & $7.0 \pm 0.5$ \\
Kalirin $(n=9)$ & $12 \pm 2.2^{a}$ & $25 \pm 5^{a}$ & $69 \pm 14^{a}$ \\
KalGEF1 $(n=14)$ & $18 \pm 1^{b}$ & $36 \pm 4^{b, c}$ & $91 \pm 10^{b}$ \\
\hline
\end{tabular}

Data from Neurolucida morphometric analyses represent the mean \pm SEM.

* Measured within $300 \mu \mathrm{m}$ radius of cell.

${ }^{a}$ Different from control, $p<0.001$.

${ }^{b}$ Different from control and kalirin, $p<0.001$.

${ }^{c}$ Different from kalirin, $p<0.02$.

creased the number of somal fiber outgrowths more than sixfold in 50 and $75 \%$ of the epitope-expressing neurons, respectively (Table 1). The appearance of new fibers $24 \mathrm{hr}$ after plasmid microinjection was distinguished readily by the presence of brightly fluorescent growth cones at the distal tips of fibers emerging from the soma; these were distinguished easily from the very much longer preexisting primary fibers.

The Kalirin-induced fibers were highly dynamic. After initiation, the new fibers attained $100-500 \mu \mathrm{m}$ in length within $24 \mathrm{hr}$, which indicated an average initial extension rate that exceeded 10 $\mu \mathrm{m} / \mathrm{hr}$. The rate of fiber growth was not affected by the number of newly formed fibers. Fiber branching, as assessed by either fiber segment or node analyses, increased $\sim 10$-fold compared with control (Table 1), and fiber directionality changed frequently. Consistent with this observation, time-lapse photomicroscopy revealed substantial growth cone motility. As anticipated, the neuronal phenotypic profiles, observed $72 \mathrm{hr}$ after injection with Kalirin-12 and Kalirin-9 expression vectors, revealed significant diversity that may reflect differences in level of Kalirin expression as well as the functional interactions of the different Kalirin domains in different neurons. No changes in the preexisting principal fibers were noted in the microinjected cells. The duration of the experimental observations was limited only by the lifetime of expression of the EGFP.

\section{Kalirin-9 and Kalirin-12 are expressed in sympathetic SCG neurons}

We used an antiserum to the spectrin-like region of Kalirin to identify the forms and levels of Kalirin in extracts of postnatal day 2 (P2) SCG or cultured SCG neurons (Fig. 3); extracts of adult rat cerebral cortex were analyzed for comparison. Western blot analysis identified Kalirin-12 (470 kDa) and Kalirin-9 (370 kDa) (Fig. 3A). Kalirin-9 is slightly more abundant than Kalirin12 , and Kalirin levels in the P2 ganglia are approximately onehalf of the levels in adult cortex. Western blot analysis of the same extracts with an antiserum specific for the $\mathrm{C}$ terminus of Kalirin-12 confirmed the identity of the $470 \mathrm{kDa}$ protein (Fig. $3 A$, right). Kalirin-7 (190 kDa), the most abundant Kalirin isoform in adult cortex and hippocampus, is not prevalent in the cultured SCG neurons.

To evaluate the contribution of Kalirin to SCG function, we developed an antiserum to the $\mathrm{C}$ terminus of Trio and used recombinant Kalirin and Trio to ensure that our Kalirin-12 and Trio antisera had similar sensitivities. Both Kalirin-12 and Trio could be detected in extracts of P1 SCG, with levels of Kalirin-12 slightly greater than levels of Trio (Fig. 3B); taking into account the existence of multiple Kalirin isoforms, it is clear that SCG Kalirin protein levels exceed those of Trio. We also used metabolic labeling followed by immunoprecipitation and fluorography

\section{A. Kalirin Expression}
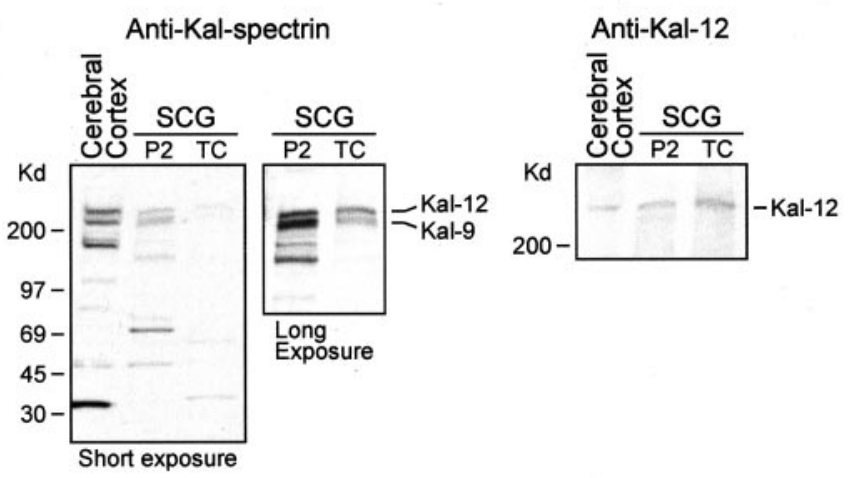

B. Kalirin and Trio Expression

C. Metabolic Labeling
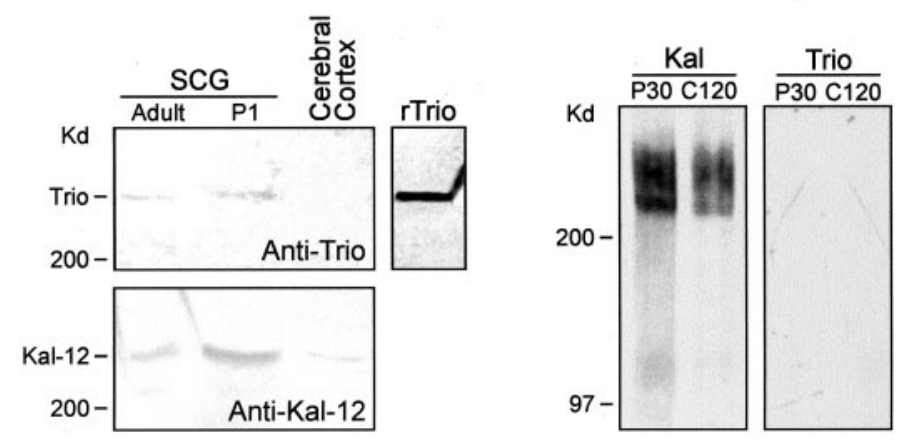

Figure 3. SCG contains more Kalirin than Trio. A, Detergent extracts (30 $\mu \mathrm{g}$ of total protein) of adult rat cerebral cortex, P2 SCG, or $10 \mathrm{~d}$ SCG cultures $(T C)$ were fractionated on $4-15 \%$ gradient gels, transferred to polyvinylidene difluoride (PVDF) membranes, and visualized by Western blot analysis by using kal-spectrin antibody and enhanced chemiluminescent reagents; a longer exposure is shown also. The bands $<200 \mathrm{kDa}$ were not visualized by Kalirin-7-specific antibodies (Penzes et al., 2001). Molecular weight markers are indicated on the left. The same samples were visualized with an antiserum selective for the $\mathrm{COOH}$ terminus of Kalirin-12. B, Detergent extracts (30 $\mu \mathrm{g}$ of total protein) of adult or P1 SCG and adult rat cerebral cortex were fractionated on $5 \%$ gels and visualized by Western blot analysis with either the Kalirin- 12 or Trio antibody; an aliquot of recombinant Trio was analyzed separately to demonstrate recovery and verify its molecular mass. The concentrations of the Kalirin-12 and Trio antibodies were chosen to yield equivalent signals when equimolar amounts of recombinant kalKinase and TrioKinase were analyzed. $C$, SCG neurons were incubated in defined serumfree medium containing $\left[{ }^{35} \mathrm{~S}\right] \mathrm{Met}$ for $30 \mathrm{~min}$ and harvested (pulse, $30 \mathrm{~min}$; P30) or chased for an additional $120 \mathrm{~min}$ (chase, $120 \mathrm{~min}$; C120) in serum-free medium. Cultures were extracted and immunoprecipitated with antibody to kal-spectrin ( Kal) or Trio (Trio). After isolation with protein A-Sepharose beads, the samples were fractionated on $5 \%$ gels for fluorography (6d).

to compare expression of the two proteins. Although synthesis of Kalirin-12 and Kalirin-9 was readily apparent, synthesis of Trio was not detectable in the same extracts (Fig. 3C). From these biochemical assessments Kalirin appeared to be the more physiologically relevant protein in sympathetic neurons.

\section{Kalirin GEF1 domain is responsible for the induction of fiber sprouting}

To evaluate whether one particular catalytic region of Kalirin was able to drive the observed fiber initiation phenotype, we microinjected expression constructs for kalGEF1, kalGEF2, or Kalirin kinase (kalKinase) into the sympathetic neurons along with the EGFP expression vector (Fig. 4). The kalGEF1 domain alone consistently produced the dramatic neuronal fiber outgrowth of 
Figure 4. KalGEF1 domain expression is key to the Kalirin-induced fiber outgrowth phenotype. $A$, Microinjection of sympathetic neuron with $200 \mathrm{ng} / \mu \mathrm{l} \mathrm{kal-}$ GEF1 expression vector induced robust fiber outgrowth and branching (compare with Fig. $2 A$ ). Growth cones at distal fiber terminals appeared as broad lamellipodial sheets (arrowheads). B, $C$, A neuron injected with kalGEF1 and EGFP $(B)$ and processed immunocytochemically to visualize the myc-epitope of the kalGEF1construct $(C)$ demonstrated kalGEF1 expression in newly formed fibers and terminals. $D, E$, KalGEF1induced fiber initiation resulted from extensive actin cytoskeleton reorganization. Micrographs of two different kalGEF1/EGFP-injected neurons ( green) were merged with micrographs of the same neurons visualized with TRITCphalloidin (red). The noninjected sympathetic neurons $(D$, asterisk) displayed relatively uniform staining for filamentous actin. Expression of kalGEF1 caused a redistribution of filamentous actin to emerging fiber outgrowths $(D$; arrowheads mark red lamellipodial filigree emerging from green microinjected neuron) and to large aggregates in the perinuclear region of the cell soma (yellow represents actin aggregates from green and red fluorescence overlay). At later stages of fi-
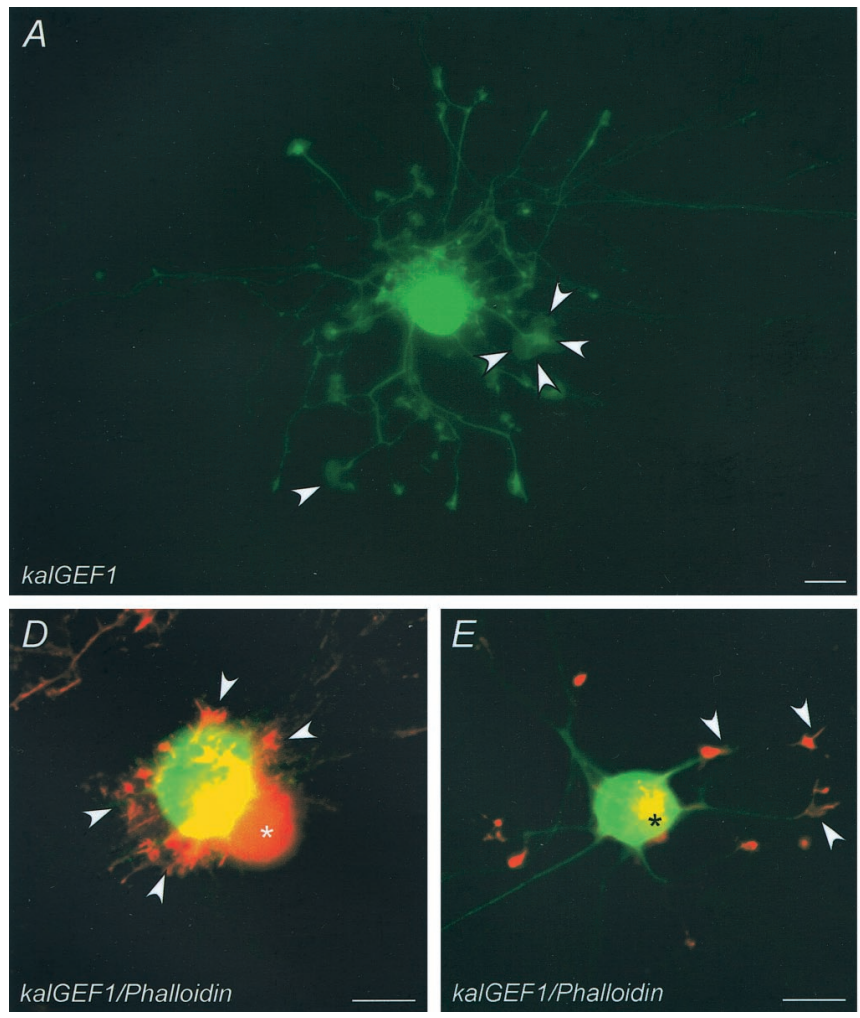
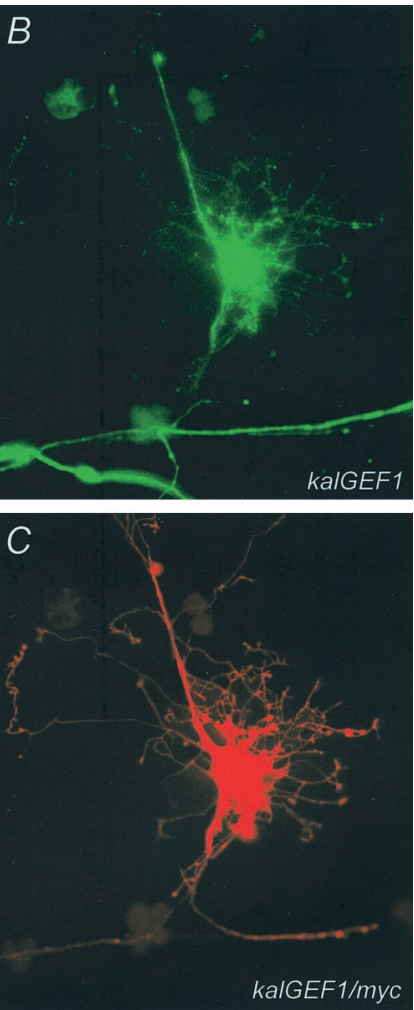

ber development the kalGEF1-injected neurons typically displayed prominent staining for filamentous actin (red) at growth cones (E, arrowheads); aggregates of filamentous actin were still apparent in neuronal soma (asterisk).
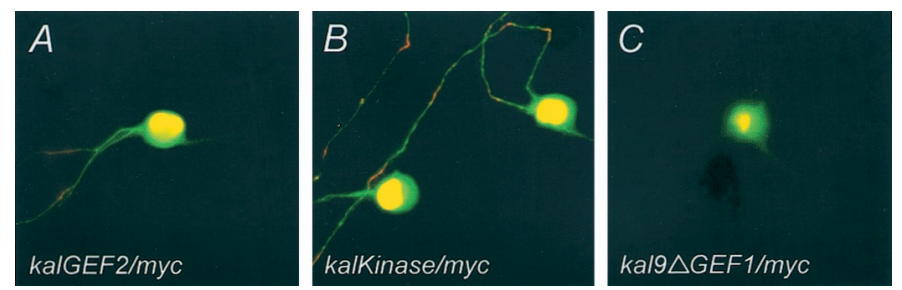

Figure 5. Kalirin GEF2 and kinase domains do not induce fiber outgrowth. Microinjection of kalGEF2, kalKinase, or kal9 $\mathrm{GEF} 1$ did not induce changes in sympathetic neuronal phenotype. $A$, A neuron injected with kalGEF2 and EGFP was processed immunocytochemically to visualize the myc-epitope of the kalGEF2 construct; the EGFP image ( green) was merged with the $\mathrm{Cy} 3$ image (red) for the epitope (yellow, from green and red Cy3 overlay). No changes in phenotype were noted in kalGEF2expressing neurons when compared with control despite high levels of myc-expression. B, Two kalKinase/EGFP-injected neurons (green) with high levels of myc-epitope expression (yellow) failed to demonstrate an outgrowth phenotype. $C$, Similarly, expression of a Kalirin-9 GEF1 deletion construct in sympathetic neurons did not elicit the fiber initiation phenotype despite high myc-expression levels ( yellow). The patchy staining for kalKinase in the processes was a reproducible observation. Scale bars, $25 \mu \mathrm{m}$.

the Kalirin phenotype in $>90 \%$ of the injected neurons (Fig. $4 A$ ). Neither kalGEF2 nor kalKinase expression organized new somal fiber outgrowths despite the demonstrated high expression of each protein (Fig. 5A,B). Microinjection of SCG neurons with Kalirin constructs with a kalGEF1 deletion (Fig. $5 C$; kal9 $\Delta$ GEF1) also failed to elicit the characteristic outgrowth phenotype, indicating that the neuronal fiber initiation response was dependent on the GEF1 domain.

The kalGEF1 phenotypic response was robust but not identical to that for Kalirin-9 or Kalirin-12. Unlike the Kalirin-induced fiber outgrowths, which exhibited tapered growth cones at their terminals, the growth cones at the terminals of kalGEF1-induced processes terminated in broad lamellipodial sheets (Fig. 4A). These large lamellipodial structures at the advancing fiber terminals resisted condensation during fiber elongation and retained their broad lamellipodial features until the fibers migrated significant distances from the soma. Expression of the kalGEF1 domain induced a ninefold increase in somal fiber outgrowth compared with control (Table 1). Staining for the myc-epitope revealed kalGEF1 expression not only in the soma but also in the newly formed fibers and growth cones, suggesting that kalGEF1 participates in local cytoskeletal restructuring for the outgrowth process (Fig. 4B,C). Consistent with these ideas, the kalGEF1induced changes were accompanied by striking cellular actin cytoskeleton reorganization; the bulk of the cellular and cortical actin adjacent to the plasma membrane was redistributed to the newly formed lamellipodial fiber structures (Fig. 4D,E). This redistribution was so extensive that the filamentous actin staining in the soma proper was almost eliminated. These studies demonstrate that kalGEF1 activity is coupled to actin reassembly processes that are necessary for neuronal fiber initiation and outgrowth.

By collecting sequential photomicrographs to evaluate fiber growth, we deduced that the kalGEF1-induced fibers presented a structured delayed mode of branching, characterized by the protrusion of collateral fibers and growth cones at specific regions of cytoskeleton instability along the advancing fiber. The sites of branching were identified first by the appearance of lamellipodial and filopodial activity. This is one preferential mode described for axon branching and is distinct from growth cone splitting mechanisms more characteristic of dendrites (Acebes and Ferrús, 2000). The initiation of new fiber formation from the soma was 

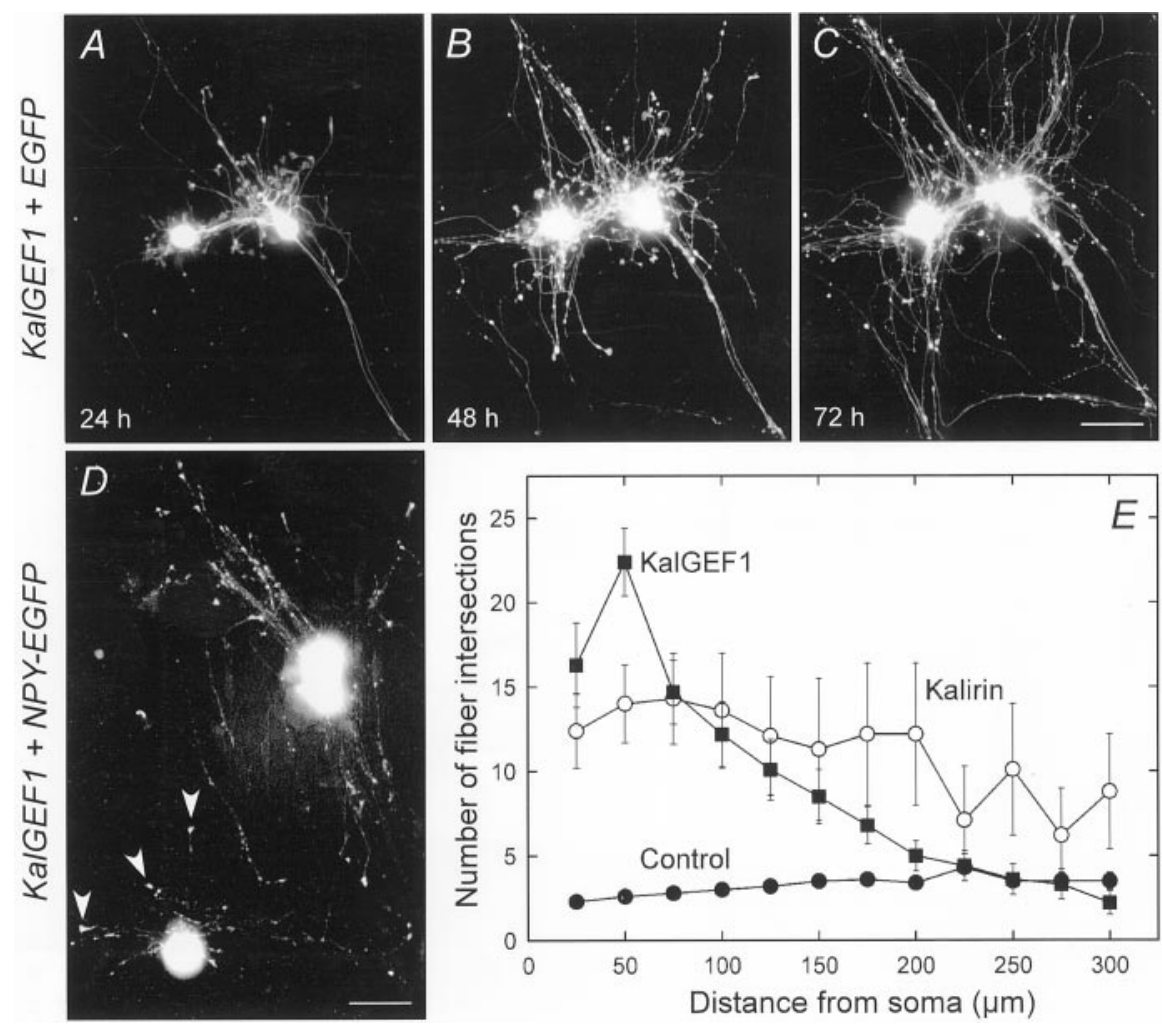

Figure 6. KalGEF1-induced fiber extensions are axons. $A-C$, Two sympathetic neurons microinjected with kalGEF1 and EGFP were photographed 24, 48, and $72 \mathrm{hr}$ after injection. Fibers initiated after kalGEF1 expression extended rapidly over time. Note the continued emergence of some new fibers even 48 and $72 \mathrm{hr}$ after injection. $D$, KalGEF1-induced fibers contained neuropeptide vesicles. Neurons were coinjected with kalGEF1 and NPY-EGFP constructs. Secretory granules containing the NPY-EGFP fusion protein were visualized on the basis of the localization of EGFP; proteins were routed from cellular sites of biosynthesis to growth cones of newly formed fibers (arrowheads; punctate green fluorescent endings). $E$, Sholl analysis of Kalirin-initiated fibers and kalGEF1-initiated fibers 2-3 d after microinjection. Control EGFP-injected neurons (CTL; $n=24$ ) demonstrated a slight increase in fiber crossings at distant intervals from the branching of principal fibers. Fiber outgrowths from kalGEF1-injected neurons $(n=14)$ produced a ninefold increase in fiber crossings ( $50 \mu \mathrm{m}$ distance) that diminished to control levels as the Sholl radii exceeded the lengths of the newly formed fibers. Post hoc Student-NewmanKeuls analyses revealed significant differences from CTL at $25-175 \mu \mathrm{m}$ distances $(p<0.001)$. Kalirininjected neurons produced a sixfold increase in intersections $(n=9)$. Fiber lengths were longer than kalGEF1-injected neurons but variable; differences in the number of fiber crossings compared with control were significant for all radial points $(p<$ 0.001). Data are mean \pm SEM, from counting fiber intersections for many neurons. Scale bars, $50 \mu \mathrm{m}$. often continuous; as newly formed fibers extended in length, new lamellipodial structures appeared around the soma with the subsequent appearance of neurites (Fig. 6A-C).

\section{The new fibers induced by kalGEF1 are axons}

We wanted to determine whether the newly initiated fibers exhibited axonal or dendritic characteristics. The kalGEF1-induced processes were uniform in diameter and exhibited both tau and paired helical filament protein immunoreactivity, suggesting that the fibers were axonal (data not shown). However, as a more rigorous demonstration of their functionality and axonal properties, the fibers and terminal growth cones of kalGEF1-injected neurons were examined for their abilities to transport and store neuropeptides. For this purpose the kalGEF1 expression vector was coinjected into sympathetic neurons with a neuropeptide Y-EGFP (NPY-EGFP) construct shown previously to yield a NPY-EGFP fusion protein that is stored in regulated secretory granules (El Meskini et al., 2001). Under these experimental conditions the soma and fibers of the kalGEF1-injected neurons were visualized solely by localizing NPY-EGFP. Like before, control neurons injected with the NPY-EGFP construct alone had only one to two processes. In the kalGEF1-coexpressing cells, NPY-EGFP was localized to profuse bright punctate structures prominent in the soma and distal terminals of the newly formed fibers (Fig. 6D). Further, these punctate structures migrated during fiber extension in culture. Thus, consistent with the morphological and biochemical criteria, the kalGEF1-induced fibers demonstrated the ability to transport peptide-containing granules for storage and regulated release at their terminals, a principal defining feature of neuronal axons.

Fiber-branching frequency, assessed by enumerating the number of nodes within a $300 \mu \mathrm{m}$ radius, was also greater in kalGEF1injected neurons than in Kalirin-injected neurons (18-fold vs 12-fold for kalGEF1 and Kalirin, respectively; Table 1). The resulting increase in fiber complexity was demonstrated directly by Sholl analyses (Fig. 6E). Because new fiber length and branching frequency and pattern between Kalirin-9-injected neurons and Kalirin-2-injected neurons were not statistically different, the data sets from the two constructs were combined (Table 1; Fig. $6 E)$. When we counted fiber intersections at $25 \mu \mathrm{m}$ concentric circles from the soma, the number of fiber crossings at the proximal regions of the kalGEF1-injected neurons was sevenfold higher than control, which reflected high fiber density contributed by both new somal fiber outgrowths and branches. Because the Sholl radii exceeded the length of the newly formed kalGEF1induced fibers $(>175 \mu \mathrm{m})$, the number of intersections declined to control values represented by the lengths of the principal fibers. As evident from the Sholl analysis, although Kalirin-induced fibers and branches were fewer in number, the fibers were frequently longer (Fig. 6E).

\section{RhoG mediates the kalGEF1-induced sprouting of new axons}

The formation of lamellipodia, filopodia, and stress fibers regulated by the Rho small GTPase family members Rac1, Cdc42, and RhoA in fibroblasts bears significant similarities to advancing growth cone structures. The same Rho GTPases clearly participate in neuronal morphogenesis (Hall, 1998; Luo, 2000); Rac1, RhoA, Cdc42, and RhoG are known to affect neuronal process development, and all four Rho GTPases are expressed in the SCG (see below). To determine whether any of these Rho GTPase mediated the dramatic fiber initiation phenotype that was observed, we first tested the ability of constitutively active forms of each Rho GTPase to mimic the kalGEF1-induced neuronal outgrowth, and then we examined the ability of the corresponding dominant-negative variant to block or attenuate the kalGEF1phenotypic response.

Unexpectedly, the microinjection of vectors encoding constitu- 


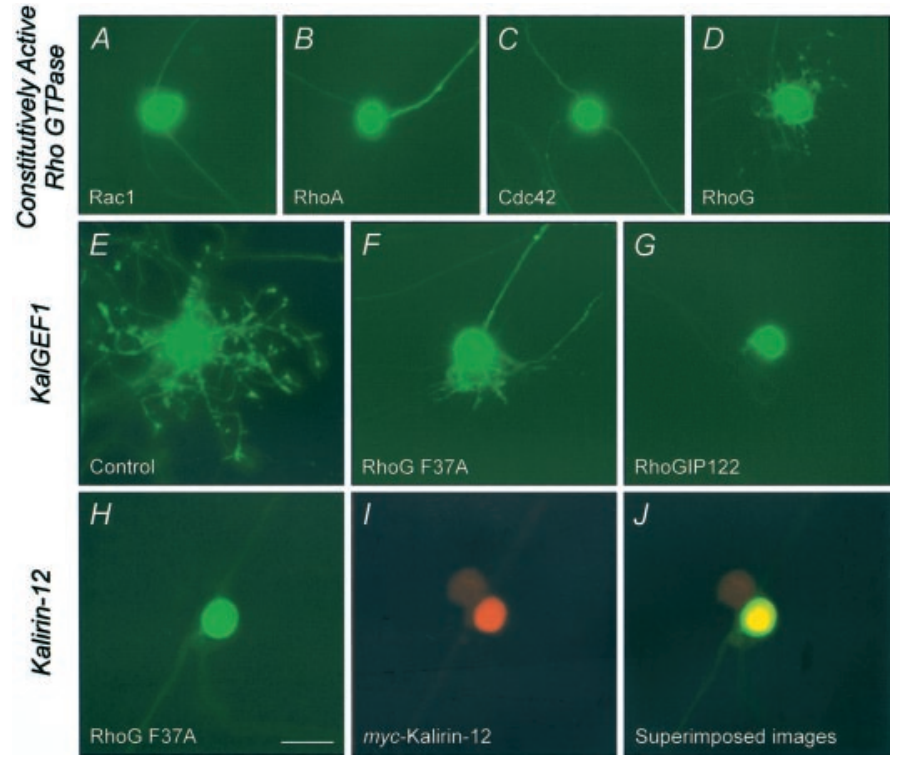

Figure 7. Kalirin-induced neuronal fiber outgrowth is mediated by RhoG. $A-D$, Constitutively active Rho GTPases (Rac1-Q61L, RhoAQ62L, Cdc42-Q61L, and Rho G12V; all at $200 \mathrm{ng} / \mu \mathrm{l}$ ) were microinjected, and the neurons were examined $48 \mathrm{hr}$ later. Only the microinjection of the active RhoG G12V induced the fiber outgrowth phenotype resembling that observed for Kalirin or kalGEF1. $E-G$, Coinjection of neurons with kalGEF1 $(50 \mathrm{ng} / \mu \mathrm{l})$ and the RhoG competitive inhibitor RhoG F37A $(F$; $200 \mathrm{ng} / \mu \mathrm{l})$ or RhoGIP122, a RhoG-GTP binding protein $(G ; 200 \mathrm{ng} / \mu \mathrm{l})$, either diminished or blocked, respectively, the fiber outgrowth phenotype observed for kalGEF1 alone $(E)$; the cells were photographed $48 \mathrm{hr}$ after injection. $H-J$, RhoG F37A expression also blocked Kalirin-12-induced fiber outgrowth. RhoG F37A fluorescence $(H$; EGFP, green) and mycepitope staining for Kalirin-12 (I; Cy3, red) are merged in $J$; new fiber extensions were never observed despite high levels of myc-expression. Scale bar, $50 \mu \mathrm{m}$.

tively active variants of Rac1, RhoA, or Cdc42 GTPases had no apparent modulatory effects on fiber initiation or on the formation of lamellipodia and/or filopodia from the soma of sympathetic neurons (Fig. 7). The soma of a very small fraction of the constitutively active Rac1-injected neurons $(\sim 10 \%)$ displayed a modest spreading phenotype reminiscent of Rac1-induced lamellipodia expansion in fibroblast lines; the few principal fibers in the injected neurons did not appear different from controls after the microinjection of constitutively active Rac1-Q61L, RhoA-Q62L, or Cdc42-Q61L (Fig. 7A-C). In contrast, the microinjection of vector encoding constitutively active RhoG G12V recapitulated the kalGEF1 neuronal outgrowth initiation phenotype (Fig. 7D). Numerous new fibers sprouted from the soma, with small tapered growth cones at the advancing distal terminals. Although the response to RhoG G12V was not as robust as that of kalGEF1, these results suggested that the fiber initiation phenotype might require RhoG activation.

Two approaches were used to test this possibility directly. First, using a minimal effective concentration of kalGEF1 to elicit the outgrowth phenotype in $90 \%$ of the injected sympathetic neurons, we coinjected a fourfold excess of vector encoding RhoG F37A, which behaves as a competitive inhibitor for endogenous RhoG (Fig. 7E vs $F$ ) (Blangy et al., 2000). The kalGEF1 phenotype was attenuated drastically. Even after extended culture periods in which cells expressing kalGEF1 alone demonstrated increased fiber number and length, neurons coinjected with kalGEF1 and RhoG F37A exhibited sharply lower levels of fiber initiation. Second, we used a recently identified RhoG binding protein (RhoGIP122) specifically to trap and remove activated GTP-bound RhoG (Blangy et al., 2000). With the same experimental paradigm, coinjection of RhoGIP122 constructs blocked completely all new kalGEF1-induced fiber outgrowths (Fig. $7 E$ vs $G)$. To determine whether fiber outgrowth induced by the fulllength Kalirin constructs was a consequence of RhoG activation, we coinjected Kalirin-12 and RhoG F37A vectors into sympathetic neurons (Fig. 7H). As with kalGEF1, RhoG F37A abrogated completely all new Kalirin-12 induced outgrowth despite high epitope expression from the Kalirin-12 vector (Fig. $7 H-J$ ). Together, these data suggest that RhoG activation is necessary for Kalirin-induced fiber outgrowth initiation.

By contrast, coinjection of kalGEF1 with dominant-negative constructs for other Rho GTPases failed to attenuate the outgrowth initiation phenotype (data not shown). Coinjection of kalGEF1 with the dominant-negative (DN) Rac1 T17N did not prevent the induction of kalGEF1-mediated fiber outgrowth. However, the distal terminals of the newly formed fibers were devoid of the conspicuous broad lamellipodial growth cones that were observed in the kalGEF1-injected neurons. Within a short period of time the kalGEF1-mediated outgrowths in the presence of DN Rac1 T17N underwent retraction, an atypical outcome of the kalGEF1 phenotype. Similarly, coinjections of DN Cdc42 T17N or DN RhoA T19N with kalGEF1 did not abrogate fiber initiation or branching. Unlike the responses with DN Rac1 T17N, kalGEF1 in the presence of DN Cdc42 or DN RhoA still initiated lamellipodial growth cones at the fiber terminals; the ensuing phenotype, especially in the presence of DN Cdc42, appeared weaker most likely because of diminished growth rates, suggesting that full expression for the Kalirin or kalGEF1 phenotype is dependent on the organized collaboration of multiple Rho GTPases.

\section{RhoG is present in SCG and binds to KalGEF1}

These results implicate RhoG or a close relative in Kalirininduced axonal initiation and growth. The ability for kalGEF1 to activate RhoG was evaluated directly in Rho GTPase binding and activity assays. Rho GTPase binding to kalGEF1 was sensitive to magnesium concentrations, and, under optimal conditions, kalGEF1 demonstrated significant binding to Rac1 and RhoG (Fig. $8 A$ ); binding to $\mathrm{Cdc} 42$, RhoA, and Ras was extremely weak, as reported for TrioGEF1 (Debant et al., 1996). When the data were normalized to Rho GTPase protein levels, kalGEF1 binding to RhoG was threefold greater than to Rac1 (Fig. 8A, bottom panel).

We used the fact that RhoGIP122 interacts specifically with activated RhoG to assess the ability of kalGEF1 to activate RhoG (Fig. 8B). Fibroblasts (HEK-293), which do not contain high levels of RhoG, were transiently transfected with RhoG in the absence or presence of kalGEF1. RhoGIP122 resin was incubated with extract, and cellular-activated RhoG bound to RhoGIP122 was visualized by Western blot analysis via the antibody to RhoG. As shown, kalGEF1 is a potent activator of RhoG (Fig. 8B).

Because the mixture of Rho proteins expressed in a given cell type is a critical determinant of its ability to respond to a particular GEF, we evaluated the expression of Rac1, RhoA, Cdc42, and RhoG in SCG, adult cortex, and liver. Sympathetic neurons express all four of these Rho GTPases (Fig. 8C). Although Rac1, RhoA, and $\mathrm{Cdc} 42$ are expressed at similar levels in adult cortex and SCG, it is notable that RhoG is not present at high levels in adult cortex. The tissue-specific expression of RhoG and other 
A. KalGEF1 Binding
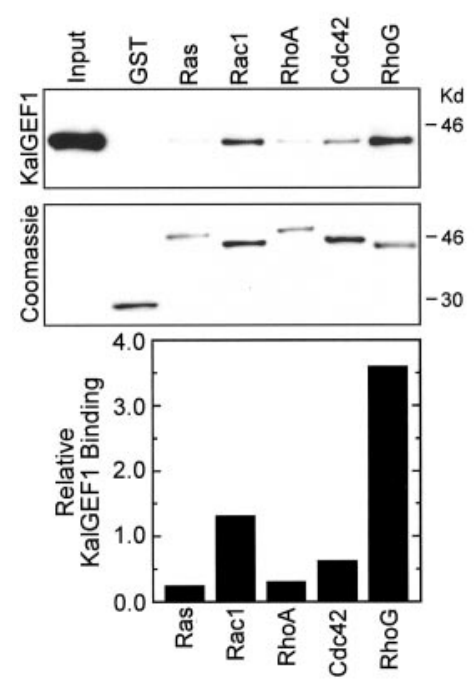

B. RhoG Activation Assay

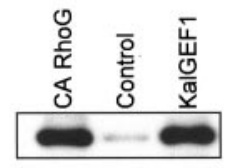

C. RhoGTPase Expression

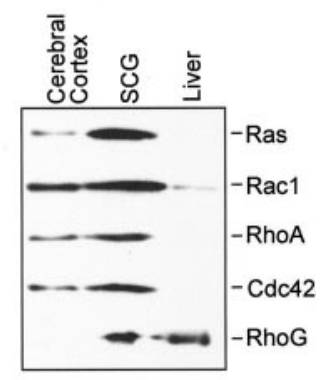

Figure 8. SCG contains RhoG, which binds to KalGEF1. $A$, Binding of kalGEF1 to nucleotide-depleted Rho proteins. KalGEF1 expressed transiently in pEAK Rapid HEK-293 cells was extracted for use in binding studies. Purified GST-Rho fusion proteins were bound to glutathione agarose beads to which aliquots of kalGEF1-containing extracts were applied. The bound fraction, representing $40 \times$ as much material as the input sample, was visualized via its $\mathrm{NH}_{2}$-terminal myc-epitope. The bound GST fusion proteins were visualized by Coomassie staining. Shown are representative data from four independent experiments. The data from $A$ were quantified, and the ratio of bound kalGEF1 to GST-Rho protein is plotted (bottom panel). B, RhoG activation assay. GSTRhoGIP $(10 \mu \mathrm{g})$ immobilized to glutathione-Sepharose beads $(25 \mu \mathrm{l})$ was incubated with extracts from cells expressing EGFP-RhoG G12V ( $C A$ $R h o G$ ) and EGFP, EGFP-RhoG and EGFP (Control), and EGFP-RhoG and KalGEF1 (KalGEF1). After being washed, the sample was analyzed by Western blot analysis, using a RhoG antiserum. $C$, Rho family members in SCG. Detergent extracts (30 $\mu \mathrm{g}$ of total protein) of adult rat cerebral cortex, P2 SCG, and adult rat liver were fractionated on $4-15 \%$ gels; the proteins were transferred to PVDF membranes and visualized by Western blot analysis by using antisera to the indicated Rho family members. Some Rho subfamilies are similar, and the specificity of the RhoA antibody was not evaluated in these tissues.

Rho family members may account for some of the differences observed in Kalirin-induced responses in central versus peripheral neurons. More recent experiments also have demonstrated that Kalirin-9 and Kalirin-12 bind to RhoG (data not shown). Together, the expression of Kalirin and Rho GTPases, especially RhoG, in peripheral sympathetic neurons and the ability of kalGEF1 to stimulate RhoG activity provide a physiological mechanism for Kalirin-mediated neuronal fiber outgrowth.

\section{Kalirin antisense oligonucleotides block normal neuronal fiber development}

The data establish the ability of exogenous KalGEF1 to initiate axonal sprouting mediated by RhoG. To determine whether endogenous Kalirin plays a role in fiber outgrowth, we microinjected a series of antisense oligonucleotides into primary sympathetic neurons shortly after initial plating. In several independent experiments the antisense oligonucleotides caused significant alterations in normal fiber outgrowth. Sympathetic neuronal fibers typically demonstrate rapid outgrowth rates; however, in neurons that were microinjected with the Kalirin antisense oligonucleotides, the growth cones became static, resulting in a failure of the axonal processes to extend over time (Fig. 9A,B). The example shown is one of 21 cases of axon growth arrest or slight axonal retraction observed after the Kalirin antisense oligonucleotides had been injected; 63 cells in total were followed after antisense oligonucleotide injection. By contrast, 12 cells were followed for $3 \mathrm{~d}$ after the injection of scrambled control oligonucleotides, and none showed any axon growth arrest, exhibiting instead continuous growth like the control GFP-injected neurons shown in Figures $2 A$ and $7 A-C$. As another control, Kalirin antisense oligonucleotides were injected along with the constitutively active RhoG and did not abrogate the GTPase-initiated fiber outgrowth (Fig. 9C).

A small number of sympathetic neurons under the culture conditions that were used displayed dendrites as identified by microtubule-associated protein (MAP2) staining (Fig. 10, inset). Introduction of Kalirin antisense oligonucleotides into these neurons not only arrested dendritic development but caused a partial retraction of existing dendrites (Fig. 10A-D). The examples shown are two of nine examples of dendritic retraction seen after injection of the Kalirin antisense oligonucleotide. The mean ratio of dendritic length for 48 to $24 \mathrm{hr}$ or 72 to $48 \mathrm{hr}$ was $0.21 \pm 0.08$ (SD), documenting a dramatic collapse of dendrites after Kalirin antisense oligonucleotide injection. The 12 cells injected with the scrambled oligonucleotides showed no dendritic collapse and continued growth as in Figures 2 and 7.

Because neuronal growth cone motility and fiber extension require cytoskeletal actin reorganization, these results suggest that sustained Kalirin expression may be essential to initiate and maintain neuronal fiber development.

\section{DISCUSSION}

The development and geometry of neuronal fibers are main determinants of the functional connectivity and complexity of the nervous system. Axonal and dendritic outgrowth, elongation, and guidance require receptor translation of diverse extracellular cues to affect both cytoskeleton reorganization and membrane biogenesis. GEF-facilitated GDP/GTP exchange on Rho GTPases is a critical component of the complex interplay of regulatory mechanisms that guide these processes. The human genome encodes at least 46 GEFs for 18 Rho GTPases, and the specific Rho GTPases, GEFs, and regulatory mechanisms that drive neuronal outgrowth responses in a physiological context are not well understood. For example, ephexins are Rho GEFs that bind EphA4 receptors; interaction of EphA4 receptors with their cell surface ligands then modulates the ability of ephexin to act as an exchange factor (Shamah et al., 2001). GEFs that are components of large multifunctional proteins may accommodate more complex regulation and coordinate the expression of more elaborate phenotypic responses to specific demands at localized cellular sites.

Kalirin and Trio are large multidomain proteins that appear to fulfill a number of important criteria for these functions. They not only contain two distinct GEF domains that activate key Rho GTPases but also possess multiple protein/protein and protein/ lipid binding motifs that could integrate the necessary signaling elements for local actin assembly at precise cellular sites. It is not yet clear whether the various isoforms of Kalirin and Trio have complementary or parallel functions during nervous system development. Unlike Trio, which is expressed in both nervous and peripheral tissues, Kalirin is expressed preferentially in the nervous system. Mice unable to express Trio display only modest alterations in nervous system organization (O'Brien et al., 2000). In contrast, Drosophila and C. elegans lacking their only Trio/ 
Figure 9. Endogenous Kalirin modulates sympathetic axonal fiber development. Sympathetic axonal fiber extension was arrested in neurons microinjected with Kalirin antisense oligonucleotides; fibers and terminals appeared static over time $(A, B)$. Of 63 neurons that followed after Kalirin antisense oligonucleotide injection, 21 neurons showed axon growth arrest or slight axonal retraction (as in $A, B$ ). In contrast, none of the 12 neurons in-
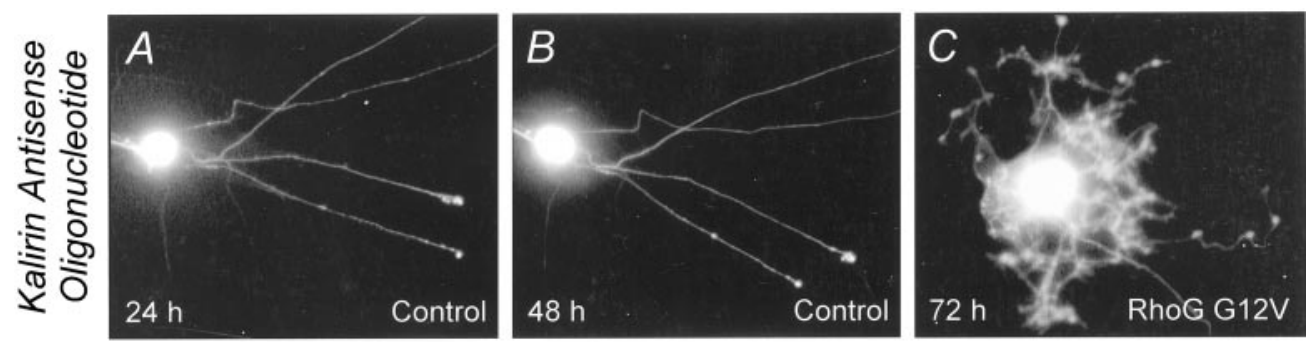

jected with scrambled control oligonucleotides showed growth arrest, instead growing steadily like the neurons in Figures $2 A$ and $7 A-C$. Kalirin antisense oligonucleotides had no effect on neuronal survival and did not impede constitutively active RhoG-induced neuronal outgrowth ( $C$ ).
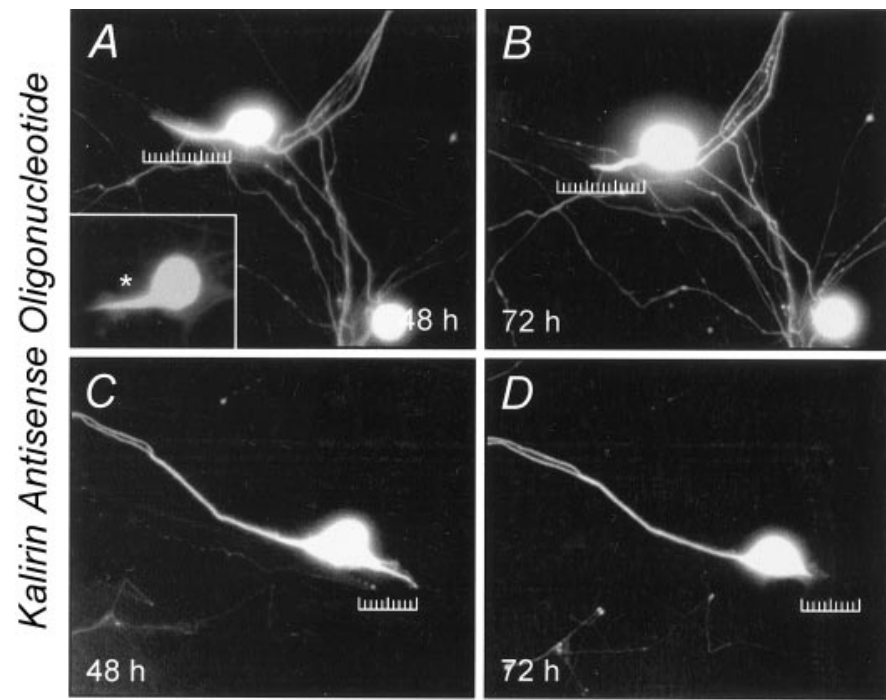

Figure 10. Endogenous Kalirin maintains dendritic outgrowth and development. A small population of sympathetic neurons developed dendrites identified by MAP2 staining via a Cy3-conjugated secondary antibody $(A$, inset; asterisk). Characteristic neuronal dendrites ceased further development and demonstrated retraction after injection with Kalirin antisense oligonucleotides (compare $A, B$ with $C, D$ ). Data are representative of the nine dendritic profiles that were examined, all of which retracted with a Kalirin antisense oligonucleotide injection. Scale bar (major tick): $15 \mu \mathrm{m}$.

Kalirin family member (dTrio and UNC-73A, respectively) have a severely affected nervous system. Our antisense studies indicate that endogenous Kalirin is important in the formation of both axons and dendrites. Disruption of Kalirin expression at an early stage of fiber outgrowth compromised the development of both axons and dendrites.

Our current studies emphasize a new and different neuronal fiber outgrowth activity associated with Kalirin function. Postganglionic sympathetic neurons possess the necessary cellular elements to generate simple, long projection fibers to appropriate targets and have been well studied both in vivo and in vitro with respect to receptor-mediated mechanisms in fiber production. SCG neurons express both Kalirin and Trio, with levels of Kalirin protein in excess of levels of Trio protein. Based on these properties, these neurons afford a tenable means for evaluating Kalirin function in a physiologically relevant context. Distinct from the neuronal guidance functions described previously for dTrio, Kalirin orchestrates the genesis of new somal fibers. Kalirin-12 and Kalirin-9 produce fibers of uniform diameter with tapered growth cones with relatively long intervals between branch points; the number of new fibers and branch nodes increases more than fivefold. Once initiated, the neurites elongate rapidly, reaching lengths exceeding 300-500 $\mu \mathrm{m}$ within $72 \mathrm{hr}$ of injection; these growth rates (up to $10 \mu \mathrm{m} / \mathrm{hr}$ ) are comparable with the maximal rates described previously for hippocampal neurons (Dotti et al., 1988).

Because Kalirin-9 and Kalirin-12 induce similar outgrowth phenotypes, the kinase domain is not essential for this response. The function of each GEF domain was evaluated separately. Expression of kalGEF1 alone induces an outgrowth phenotype that is distinct from that of Kalirin-9 or Kalirin-12. As in similar studies with dTrio, kalGEF2 had no apparent effects (Newsome et al., 2000). Although Kalirin-induced neuronal outgrowth requires the KalGEF1 domain, the other domains of Kalirin clearly play essential regulatory roles. Protein localization or the ability to control activation of kalGEF1 may be critical to achieving the phenotype observed with Kalirin-9 and Kalirin-12.

Expression of the kalGEF1 resulted in the production of large lamellipodial formations and multiple fiber sprouts. Cellular filamentous actin labeled with phalloidin was translocated to aggregates in the cell body and to the leading edges of emerging lamellipodia and growth cone structures. Even after fiber elongation, the most prominent areas of filamentous actin staining were identified at distal growth cones. Although the number of newly initiated fibers and branch nodes in the kalGEF1-injected neurons was $50 \%$ greater than in neurons overexpressing Kalirin-12 or Kalirin-9, the overall length of the fibers 72 hr after injection appeared shorter than the Kalirin-9-overexpressing neurons and Kalirin-12-overexpressing neurons (Fig. 6E). Fiber length may be driven by microtubule formation and the ability of microtubules to protrude into the actin meshwork at the growth cones (Bradke and Dotti, 1999). Microtubule entry, fiber elongation, and axon formation are facilitated by growth cones with highly dynamic actin processes that produce loose meshwork structures; in contrast, growth cones with stable polymerized actin may be destined to bear dendritic characteristics. Although KalGEF2 alone had no apparent phenotype, when present in the context of Kalirin-12 or Kalirin-9, it might coordinate the regulation of actin stability and facilitate fiber elongation. Consistent with this suggestion, when expressed in neonatal cortical neurons, kalGEF2 alone promoted the extension of neurites (Penzes et al., 2001b). TrioGEF2 demonstrates selectivity for RhoA (Debant et al., 1996), and kalGEF2 or post-translationally modified forms of kalGEF2 also may exhibit differential Rho GTPase specificity.

Despite differences in overall fiber length and branching, both the kalGEF1-induced fibers and Kalirin-induced fibers were axon-like in character. The fibers demonstrated immunoreactivity for axonal markers, and time-lapse microscopy revealed an axonal mode of branching. As a more direct means of elucidating the functional character of these fibers, we expressed a chimeric 
uncleavable NPY-EGFP fusion protein in neurons expressing kalGEF1 (El Meskini et al., 2001). Sympathetic neurons express high levels of neuropeptide $\mathrm{Y}$ and, like the endogenous neuropeptide, NPY-EGFP is routed to dense core vesicles via the regulated secretory pathway (El Meskini et al., 2001). When the kalGEF1 and NPY-EGFP constructs were coinjected, the soma and distal terminals of the kalGEF1-induced fibers were labeled with the chimeric protein, indicating that vesicles were transported and stored at the new fiber terminals for potential release. These are key properties of functional fibers and suggest that the majority of the Kalirin-initiated fibers and kalGEF1-initiated fibers is axonal in character.

We demonstrated previously that kalGEF1 is an exchange factor for Rac1 (Penzes et al., 2001a). However, the initiation of neuronal outgrowth by Kalirin or kalGEF1 is not mediated directly by Rac1, RhoA, or Cdc42; instead, RhoG plays an essential role. Among the constitutively active Rho GTPases that were examined, only the activated RhoG mimicked the kalGEF1 phenotype. Conversely, dominant-negative forms of Rac1, RhoA, or Cdc42 failed to abrogate kalGEF1-induced neuronal outgrowth. In contrast, both a RhoG competitive inhibitor and a protein that binds activated RhoG were able to block the kalGEF1 and Kalirin-12 phenotypes. RhoG also was shown to be a neurite outgrowth regulator in pheochromocytoma cells (Katoh et al., 2000). Expression of RhoG in sympathetic neurons, but not in cortical neurons, may contribute to their distinctly different responses to kalGEF1.

KalGEF1 binds to and activates multiple Rho GTPases, as described previously for Vav and TrioGEF1 (Schuebel et al., 1998; Bellanger et al., 2000; Blangy et al., 2000). Although kalGEF1 binds to several Rho GTPases in in vitro assays, the ability of the RhoG competitive inhibitor and binding protein to abrogate completely any kalGEF1-mediated phenotype in sympathetic neurons supports recent suggestions that RhoG functions upstream of other Rho GTPases. Studies in fibroblasts and PC12 pheochromocytoma cells indicate that Cdc42, Rac1, and RhoA can form an interactive cascade (Nobes and Hall, 1995). Similarly, activated RhoG can result in the independent activation of Rac1 and Cdc42 (Gauthier-Rouvière et al., 1998; Blangy et al., 2000). In the physiological setting that was examined, our studies suggest that kalGEF1 does not activate multiple Rho GTPases directly; the Kalirin neuronal outgrowth phenotype appears to be initiated instead by RhoG-mediated signal transduction that coordinates multiple downstream pathways.

The distinct and differential roles of Kalirin and Trio in the nervous system are still unclear. There is a progression in Kalirin expression during neuronal development such that Kalirin becomes predominant compared with Trio at nervous system maturation (McPherson et al., 2002), yet the functional implications of that transition with respect to dendritic structure or axonal outgrowth have not been determined. Whereas the GEF1/GEF2 domains between the two proteins may display preferences for specific Rho GTPases, recent studies demonstrating TrioGEF1 binding to RhoG suggest that the GEF domains may be functionally similar. The associated domains in Kalirin and Trio may harbor differential intracellular-targeting information for each protein to organize specific fiber patterns; alternatively, Kalirin and Trio may be regulated differentially under various physiological contexts. More detailed studies may elucidate the functional distinctions.

With migration to the appropriate destination, developing neurons extend dendritic and axonal processes to adopt a character- istic morphological phenotype commensurate with their functional complexity. These processes are not well understood, but recent data have indicated that these actin cytoskeletonremodeling events involve the GEF protein activation of Rho GTPases, exemplified by studies of dTrio in axonal guidance. Distinct from axonal-pathfinding functions, we now demonstrate that Kalirin, a related Dbl-GEF protein, is capable of directing neuronal fiber initiation, elongation, and branching in a physiologically relevant context via RhoG-dependent mechanisms. This process provides a mechanism for the genesis of morphological diversity among central and peripheral neurons during development. Because neuronal phenotypic diversity forms a cellular basis for functional connectivity, this family of related proteins may be key elements in establishing neural circuitry. Because neuronal synaptic plasticity, remodeling, and regeneration also impinge on neuronal outgrowth processes, we suggest that Kalirin/Trio-related proteins also may mediate many of these adaptive mechanisms in the mature nervous system.

\section{REFERENCES}

Acebes A, Ferrús A (2000) Cellular and molecular features of axon collaterals and dendrites. Trends Neurosci 23:557-565.

Alam MR, Caldwell BD, Johnson RC, Darlington DN, Mains RE, Eipper BA (1996) Novel proteins that interact with the COOH-terminal cytosolic routing determinants of an integral membrane peptideprocessing enzyme. J Biol Chem 271:28636-28640.

Alam MR, Johnson RC, Darlington DN, Hand TA, Mains RE, Eipper BA (1997) Kalirin, a cytosolic protein with spectrin-like and GDP/ GTP exchange factor-like domains that interacts with peptidylglycine $\alpha$-amidating monooxygenase, an integral membrane peptide-processing enzyme. J Biol Chem 272:12667-12675.

Awasaki T, Saito M, Sone M, Suzuki E, Sakai R, Ito K, Hama C (2000) The Drosophila Trio plays an essential role in patterning of axons by regulating their directional extension. Neuron 26:119-131.

Bateman J, Shu H, Van Vactor D (2000) The guanine nucleotide exchange factor Trio mediates axonal development in the Drosophila embryo. Neuron 26:93-106.

Bellanger JM, Astier C, Sardet C, Ohta Y, Stossel TP, Debant A (2000) The Rac1- and RhoG-specific GEF domain of Trio targets filamin to remodel cytoskeletal actin. Nat Cell Biol 2:888-892.

Blangy A, Vignal E, Schmidt S, Debant A, Gauthier-Rouvière C, Fort P (2000) TrioGEF1 controls Rac- and Cdc42-dependent cell structures through direct activation of RhoG. J Cell Sci 133:729-739.

Braas KM, May V (1999) Pituitary adenylate cyclase-activating polypeptides directly stimulate sympathetic neuron neuropeptide Y release through $\mathrm{PAC}_{1}$ receptor isoform activation of specific intracellular signaling pathways. J Biol Chem 274:27702-27710.

Bradke F, Dotti CG (1999) The role of local actin instability in axon formation. Science 283:1931-1934.

Debant A, Serra-Pages C, Seipel K, O'Brien S, Tang M, Park SH, Streuli M (1996) The multidomain protein Trio binds the LAR transmembrane tyrosine phosphatase, contains a protein kinase domain, and has separate Rac-specific and Rho-specific guanine nucleotide exchange factor domains. Proc Natl Acad Sci USA 93:5466-5471.

Dickson BJ (2001) Rho GTPases in growth cone guidance. Curr Opin Neurobiol 11:103-110.

Dotti CG, Sullivan CA, Banker GA (1988) The establishment of polarity by hippocampal neurons in culture. J Neurosci 8:1454-1468.

El Meskini R, Jin L, Marx R, Bruzzaniti A, Lee J, Emeson RB, Mains RE (2001) A signal sequence is sufficient for green fluorescent protein to be routed to regulated secretory granules. Endocrinology 142:864-873.

Francis NJ, Landis SC (1999) Cellular and molecular determinants of sympathetic neuron development. Annu Rev Neurosci 22:541-566.

Gauthier-Rouvière C, Vignal E, Mériane M, Roux P, Montcourier P, Fort P (1998) RhoG GTPase controls a pathway that independently activates Rac1 and Cdc42Hs. Mol Biol Cell 9:1379-1394.

Gertler FB, Comer AR, Juang J-L, Ahern SM, Clark MJ, Liebl EC, Hoffmann FM (1995) enabled, a dosage-sensitive suppressor of mutations in the Drosophila Abl tyrosine kinase, encodes an Abl substrate with SH3 domain-binding properties. Genes Dev 9:521-533.

Giger RJ, Urquhart ER, Gillespie SK, Levengood DV, Ginty DD, Kolodkin AL (1998) Neuropilin-2 is a receptor for semaphorin IV: insight into the structural basis of receptor function and specificity. Neuron 21:1079-1092.

Guo X, Rueger D, Higgins D (1998) Osteogenic protein-1 and related bone morphogenetic proteins regulated dendritic growth and expression of microtubule-associated protein-2 in rat sympathetic neurons. Neurosci Lett 10:131-134. 
Hall A (1998) Rho GTPases and the actin cytoskeleton. Science 279:509-514.

Hing H, Xiao J, Harden N, Lim L, Zipursky SL (1999) Pak functions downstream of Dock to regulate photoreceptor axon guidance in Drosophila. Cell 97:853-863.

Johnson RC, Penzes P, Eipper BA, Mains RE (2000) Isoforms of kalirin, a neuronal $\mathrm{Dbl}$ family member, generated through use of different $5^{\prime}$ and 3 '-ends along with an internal translational initiation site. J Biol Chem 275:19324-19333.

Katoh H, Yasui H, Yamaguchi Y, Aoki J, Fujita H, Mori K, Negishi M (2000) Small GTPase RhoG is a key regulator for neurite outgrowth in PC12 cells. Mol Cell Biol 20:7378-7387.

Kaufmann N, Willis ZP, Van Vactor D (1998) Drosophila Rac1 controls motor axon guidance. Development 125:453-461.

Kozma R, Sarner S, Ahmed S, Lim L (1997) Rho family GTPases and neuronal growth cone remodeling: relationships between increased complexity induced by $\mathrm{Cdc} 42 \mathrm{Hs}$, Rac1, and acetylcholine and collapse induced by RhoA and lysophosphatidic acid. Mol Cell Biol 17:1201-1211.

Krueger NX, Van Vactor D, Wan H, Goodman CS, Gelbart W, Saito H (1996) The transmembrane tyrosine phosphatase DLAR controls motor axon guidance. Cell 84:611-622.

Lanier LM, Gertler FB (2000) From Abl to actin: Abl tyrosine kinase and associated protein in growth cone motility. Curr Opin Neurobiol 10:80-87.

Liebl EC, Forsthoefel DJ, Franco LS, Sample SH, Hess JE, Cowger JA, Chandler MP, Shupert AM, Seeger MA (2000) Dosage-sensitive, reciprocal genetic interactions between Abl tyrosine kinase and the putative GEF Trio reveal Trio's role in axon pathfinding. Neuron 26:107-118.

Lin MZ, Greenberg ME (2000) Orchestral maneuvers in the axon. Trio and the control of axonal guidance. Cell 101:239-242.

Luo L (2000) Rho GTPases in neuronal morphogenesis. Nat Rev Neurosci 1:173-180.

Luo L, Jan LY, Jan YN (1997) Rho family GTP-binding proteins in growth cone signaling. Curr Opin Neurobiol 7:81-86.

Ma X-M, Johnson RC, Mains RE, Eipper BA (2001) Expression of Kalirin, a neuronal GDP/GTP exchange factor of the Trio family, in the central nervous system of the adult rat. J Comp Neurol 429:388-402.

Mains RE, Alam MR, Johnson RC, Darlington DN, Back N, Hand TA Eipper BA (1999) Kalirin, a multifunctional PAM COOH-terminal domain interactor protein, affects cytoskeletal organization and ACTH secretion from AtT-20 cells. J Biol Chem 274:2929-2937.

May V, Brandenburg CA, Braas KM (1995) Differential regulation of sympathetic neuron neuropeptide $\mathrm{Y}$ and catecholamine content and secretion. J Neurosci 15:4580-4591.

McPherson CE, Eipper BA, Mains RE (2002) Genomic organization and differential expression of human and rat kalirin isoforms. Gene 284:41-51.

Milgram SL, Kho ST, Martin GV, Mains RE, Eipper BA (1997) Localization of integral membrane peptidylglycine $\alpha$-amidating monooxygenase in neuroendocrine cells. J Cell Sci 110:695-706.
Mueller BK (1999) Growth cone guidance: first steps toward a deeper understanding. Annu Rev Neurosci 22:351-388.

Newsome TP, Schmidt S, Dietzl G, Keleman K, Asling B, Debant A, Dickson BJ (2000) Trio combines with Dock to regulate Pak activity during photoreceptor axon pathfinding in. Drosophila. Cell 101:283-294

Nobes CD, Hall A (1995) Rho, Rac, and Cdc42 GTPases regulate the assembly of multimolecular focal complexes associated with actin stress fibers. Cell 81:53-62.

O’Brien SP, Seipel K, Medley QG, Bronson R, Segal R, Streuli M (2000) Skeletal muscle deformity and neuronal disorder in Trio exchange factordeficient mouse embryos. Proc Natl Acad Sci USA 97:12074-12078.

Penzes P, Johnson RC, Alam MR, Kambampati V, Mains RE, Eipper BA (2000) An isoform of Kalirin, a brain-specific GDP/GTP exchange factor, is enriched in the postsynaptic density fraction. J Biol Chem 274:6395-6403.

Penzes P, Johnson RC, Sattler R, Zhang X, Huganir RL, Kambampati V, Mains RE, Eipper BA (2001a) The neuronal Rho-GEF Kalirin-7 interacts with PDZ domain-containing proteins and regulates dendritic morphogenesis. Neuron 29:229-242.

Penzes P, Johnson RC, Kambampati V, Mains RE, Eipper BA (2001b) Distinct roles for the two Rho-GEF domains of Kalirin in regulation of neurite growth and neuronal morphology. J Neurosci 21:8426-8434.

Schuebel KE, Movilla N, Rosa JL, Bustelo XR (1998) Phosphorylationdependent and constitutive activation of Rho proteins by wild-type and oncogenic Vav-2. EMBO J 17:6608-6621.

Shamah SM, Lin MZ, Goldberg JL, Estrach S, Sahin M, Hu L, Bazalakova M, Neve RL, Corfas G, Debant A, Greenberg ME (2001) EphA receptors regulate growth cone dynamics through the novel guanine nucleotide exchange factor ephexin. Cell 105:233-244.

Steven R, Kubiseski TJ, Zheng H, Kulkarni S, Mancillas J, Morales AR, Hogue CWV, Pawson T, Culotti J (1998) UNC-73 activates the Rac GTPase and is required for cell growth cone migrations in C. elegans. Cell 92:785-795.

Suter DM, Forscher P (1998) An emerging link between cytoskeletal dynamics and cell adhesion molecules in growth cone guidance. Curr Opin Neurobiol 8:106-116.

Tessier-Lavigne M, Goodman CS (1996) The molecular biology of axon guidance. Science 274:1123-1133.

Tsui-Pierchala BA, Ginty DD (1999) Characterization of an NGF-PTrkA retrograde-signaling complex and age-dependent regulation of TrkA phosphorylation in sympathetic neurons. J Neurosci 19:8207-8218.

Van Aelst L, D'Souza-Schorey C (1997) Rho GTPases and signaling networks. Genes Dev 11:2295-2322.

Van Vactor D (1998) Tyrosine phosphatases in the nervous system. Curr Opin Cell Biol 10:174-181.

Xu X-M, Fisher DA, Zhou L, White FA, Ng S, Snider WD, Luo Y (2000) The transmembrane protein semaphorin 6A repels embryonic sympathetic axons. J Neurosci 20:2638-2648.

Zipkin ID, Kindt RM, Kenyon CJ (1997) Role of a new Rho family member in cell migration and axon guidance in C. elegans. Cell 90:883894. 